\title{
Vision Based Supervised Restricted Boltzmann Machine Helps to Actuate Novel Shape Memory Alloy Accurately
}

Ritaban Dutta ( $\square$ ritaban.dutta@csiro.au )

CSIRO DATA61

Cherry Chen

CSIRO Manufacturing

David Renshaw

CSIRO Manufacturing

Daniel Liang

CSIRO Manufacturing

\section{Research Article}

Keywords: shape memory alloys (SMAs), next-generation soft robotic systems, Restricted Boltzmann Machine, predictive generalisation

Posted Date: June 22nd, 2021

DOl: https://doi.org/10.21203/rs.3.rs-577116/v1

License: (c) (i) This work is licensed under a Creative Commons Attribution 4.0 International License. Read Full License

Version of Record: A version of this preprint was published at Scientific Reports on August 12th, 2021. See the published version at https://doi.org/10.1038/s41598-021-95939-y. 


\section{Abstract}

Extraordinary shape recovery capabilities of shape memory alloys (SMAs) have made them a crucial building block for the development of next-generation soft robotic systems and associated cognitive robotic controllers. In this study we desired to determine whether combining video data analysis techniques with machine learning techniques could develop a computer vision based predictive system to accurately predict force generated by the movement of a SMA body that is capable of a multi-point actuation performance. We identified that rapid video capture of the bending movements of a SMA body while undergoing external electrical excitements and adapting that characterisation using computer vision approach into a machine learning model, can accurately predict the amount of actuation force generated by the body. This is a fundamental area for achieving a superior control of the actuation of SMA bodies. We demonstrate that a supervised machine learning framework trained with Restricted Boltzmann Machine (RBM) inspired features extracted from 45000 digital thermal infrared video frames captured during excitement of various SMA shapes, is capable to estimate and predict force and stress with $93 \%$ global accuracy with very low false negatives and high level of predictive generalisation.

\section{Introduction}

Newly discovered SMAs are increasingly being used for application solutions in automotive [1], aerospace [2], construction and other commercial fields [3,4] for their extraordinary ability to fully recover to their original shape from an actuated shape. The unique capability to memorize the original structural shape and recovery back to that shape from an excited state, has made this type of materials a novel candidate for the future generation soft robotics and for the applications with a requirement of lightweight or miniaturised actuation [5-8]. Recovery characteristics of this SMA provide a superior level of flexibility in movement and higher degree of freedom than moving a conventional mechanical structure, especially in applications where the volume and weight of actuators are restricted.

Thermally activated SMA based actuators are the potential candidates to replace the traditional actuation systems including for applications in space stations, satellites, or planet robots [9]. Compared with the traditional space actuating sub-systems, the SMA actuators have a high power-to-weight ratio, a simple actuation mechanism, no dust particles created or no leakage of fluids, thus reducing the complexity, sizes and the weight of a actuator [10]. The thermally induced SMAs perform shape memory effect (SME) by undergoing solid-state transformations between low and high temperature phases, which are known as martensite and austenite phases, at characteristic temperatures. The shape change intrinsically occurs only during heating from martensite to austenite phases, not cooling from austenite to martensite, referring to one-way-SME, non-reversible. By applying a series of heat treatments or "training" processes, SMA can have two-way-SME with a limited degree of reverse actuation that are of little usefulness [1112], e.g. less than 20-30-degree angles for a reversable bending strip [13]. Therefore, most of the commercial SMAs are supplied only for performing a single-point, mostly one-way, actuation - i.e. onedirection and non-reversible motion at one fixed temperature. Consequently, the lack of an effective, reversable actuation ability has limited the use of the SMA actuators to very few simple applications, 
such as one-off release in QWKNUT and Low-Force Nut (LFN) [14], locking or deploying mechanisms for truss mounting [15]folding structures [16], and deployable hinges of lightweight flexible solar array [17].

The CSIRO scientists have developed a rapid solidification process that can produce Ti-Ni based SMA foils with a larger width that can be utilised for constructing actuators [18]. More importantly, the obtained SMA foils exhibit a larger reversable actuation after thermomechanical cycling. As a demonstrative example of the shape memorisation training of a SMA body, the large-degree reversable actuation of a CSIRO-produced V-shaped-NiTi SMA body is illustrated in Fig. 1. Between electrical heating on and off, the V-shaped SMA was closing and opening over a 60 degrees angle, showcasing high potential for an industrial adaptation.

This has achieved a new step towards the wider adaptation of SMA materials in various applications. However, despite the current advances in the field of reversable shape memory materials, applications of this family of novel materials remain a unique challenge due to difficulty in accurately controlling and reversibly actuating a SMA body [19]. Like any moving physical body, SMA based structures also generate force while the whole body or a part of the body moves under an external excitement. The actuation amount and speed, thus their control, are influenced by the interaction between the internal actuation force generated at the atomic scale and the resistance to the actuation that is related to bulk materials properties. As the SMA internal interaction of the forces are unique and are difficult to quantify, the characterisation of the forces and their effects on the actuation behaviours through conventional ways either experimentally or analytical modelling are difficult and time consuming [20-22].

The aim of this study was to develop a predictive modelling system in order to actuate the novel reversable SMA materials in a more controllable way. A method to rapidly and accurately measure the amount of the force generated by the SMA movement is the first essential step to control and actuate the SMA body. The second step in undertaking this reported work was to develop an intelligent modelling system for the accurate estimation of the force generated by a group of reversable-actuating SMA bodies of various shapes (as shown in Fig. 2 and Fig. 3).

A promising predictive modelling has been proposed and developed in this paper. This combines computer vision and machine learning to characterise novel SMA materials and estimate force generated by a moving SMA body under external excitement. We employed infrared digital camera to capture the video of a moving SMA body, while measuring generated force simultaneously. The measured force was used as benchmarking ground truth data for all future modelling.

The change in the relative position and shape of the SMA body compared to its original position and shape under excitement, was captured in the video frames. This dynamically changing information about shape and position were correlated with the separately measured generated force for using the proposed predictive modelling [23-31].

We chose to use vision based supervised Restricted Boltzmann Machine (RBM) approach combined with a machine learning classifier algorithm to make this estimation. Machine learning algorithm in 
classification tasks has proven to be highly effective in a wide range of applications while being free of the restrictive assumptions of other predictive systems [32]. RBM has been proven to be a very effective technique to capture unique set of features or components from video data. RBM was used as a feature extraction technique, while extracted features were used to train a machine learning classifier for developing a predictive model capable to estimate and predict the force generated by a moving SMA body. Figure 4 showcases a schematic diagram of the employed machine learning framework involving RBM and a machine learning classifier [33-39] and prediction validation of such an approach.

\section{Preparation Of The Sma And Thermal Treatment}

In this study, NiTi based shape memory alloy (SMA) foils with a width of $30 \mathrm{~mm}$ and a thickness around 80-150 $\mu \mathrm{m}$, were prepared by planar flow casting i.e. a rapid solidification process. The SMA foils were cut into $10 \mathrm{~mm}$ width and $120 \mathrm{~mm}$ long strips and were carefully inserted into the slot of a metal mould. The mould with the sample was placed inside a tube furnace, undergoing a standard annealing process at $550^{\circ} \mathrm{C}$ for $30 \mathrm{~min}$ in an argon atmosphere before quenched in water. In this study, five different shapes of SMA strips were produced. The moulds along with the shaped SMA strips are shown in Figure 1(a). As indicated by the schematic in Figure 2 (b), a centre slot $4 \mathrm{~mm}$ wide, $115 \mathrm{~mm}$ long has been cut out of the SMA strip. This is to form the electrical current path for actuating the SMA.

\section{Chemical analysis}

The SMA foils were characterized by inductively coupled plasma optical emission spectroscopy Varian 730 -ES ICP-OES (ICP-OES). Around $0.1 \mathrm{~g}$ of the sample was dissolved in a solution of $\mathrm{HNO}_{3}, \mathrm{H}_{2} \mathrm{SO}_{4}, \mathrm{HF}$ and water before the ICP-OES analysis. After calibrated by certified multi-element solutions, the analysis results showed that the SMA consisted of $49.1 \%$ of $\mathrm{Ni}$ and $50.9 \%$ of $\mathrm{Ti}$.

\section{Thermal analysis}

Differential Scanning Calorimetry (DSC) was selected to analyse transformation temperatures of the SMA samples. Around $5 \mathrm{mg}$ of SMA sample was used for the test under a nitrogen flow ( $40 \mathrm{ml} / \mathrm{min})$. A heating and cooling schedule consisting of a heating rate from $-20^{\circ} \mathrm{C}$ to $100^{\circ} \mathrm{C}$. Followed by a cooling rate from $100{ }^{\circ} \mathrm{C}$ to $-20^{\circ} \mathrm{C}$. Both heating and cooling rates both were at $10^{\circ} \mathrm{C} / \mathrm{min}$. DSC thermogram was obtained using a Mettler Toledo DSC3. The transformation temperatures were extrapolated from the DSC data through the tangential line method: Martensite start temperature $\left(M_{S}\right)$, Martensite finish temperature $\left(M_{f}\right)$, Austenite start temperature $\left(A_{s}\right)$ and Austenite finish temperature $\left(A_{f}\right)$ are 60.8, 42.33, 72.22 and 89.54 , respectively.

\section{Shape memory alloy training}

A certain thermomechanical treatment [so-called training process, shown in Figure 1(c)] was carried out on the SMA test samples: (a) deform the samples into flat strips; (b) connect each legs of the strip to a DC power supply; (c) apply a current of $5 \mathrm{~A}$ at the voltage of $2.5 \mathrm{~V}$ for 10 seconds. The temperature of the 
samples increased due to the Joule heating effect and the samples were recovered to their initial shapes; (d) turn off the current and allow it to cool down to room temperature; (e) repeat (a) to (d) steps for 30 times. After this training, the SMA samples "remembered" two status i.e. flat and shaped when the current was on and off, showing two-way shape memory effect.

\section{Video data capturing and pre-processing to study SME}

An infrared thermal camera fixed on a test rig was used to capture the change in temperature, position and shape of the SMA bodies while under electrical excitation as shown in Figure 1(b). The trained SMA flat sample is connected to a DC supply with a current of $5 \mathrm{~A}$ and a voltage of $2.5 \mathrm{~V}$, one leg is for current in and the other is for current out to ensure a close loop [Figure 2 (a) and (b)]. When the current was applied, the temperature of the SMA was increased and it started to form the trained shape. When the current is turned off, the SMA sample recovers to the original flat shape. The thermal video files were treated as a combination of static frames of size 1200 by 1200 pixels. The individual frame was compressed to 300 by 300 pixels size and converted to grayscale images (as shown in Figure 4). The quality of the captured video was kept consistent during all experimentation in that 51000 video frames of the five different moving SMA bodies were collected. Standardizing the experimental protocol was crucial to capture data of a high quality that are consistent and completely reproducible.

The difference between two consecutive video frames (DF) was used as a differential and representative information for capturing the changes in shape and position demonstrated by the SMA body under excitement. The change in shape and position was apparently the key indicator of the force generated. Each of the DF was pre-processed to extract representative features to be used in the training and testing of the predictive machine learning algorithms. Selection of a DF to be included in the final study, was determined by a significance tolerance factor, defined by the image pixel wise difference between two consecutive frames being greater than $5 \%$. This was to eliminate the repetitive video frames (without any significant changes) from the overall analysis and any potential bias that could be created by this type of repetition. Finally, 45000 DFs were selected to be included in the analysis.

RBM was used in this study for pre-processing of the video data. We found that RBM based feature representation was better suited as an encoder for this study over a conventional autoencoder, as RBM was faster to process the large volume of video frames with standard available libraries. Parameters are estimated using Stochastic Maximum Likelihood (SML), also known as Persistent Contrastive Divergence (PCD) [31-43]. We utilized RBM as a feature extraction method to reduce the very large dimension of the video frames. We found that 150 extracted components by RBM was optimum and best to explain the variance among the video frames to be classified into one of the force classes and stress classes accurately. Optimum learning rate for the RBM was determined to be at 0.412 .

\section{Force measurement}

The tester to measure the actuation force is shown in Figure 3. It is equipped with a $20 \mathrm{Kg}$ load cell. The two sample holders of the tester were connected to a DC power supply (DPD3030, Manson). The applied 
current was $7 \mathrm{~A}$ and the voltage was $5 \mathrm{~V}$.

The SMA samples were cut into a strip with width of $5 \mathrm{~mm}$ and length of $120 \mathrm{~mm}$ for force measurement. After thermally treated, shaped and trained, the SMA test samples of different shapes are flattened and placed onto the tester with both ends of SMA sample screwed onto the tester sample holders. Torque wrench was used to tighten the screws, ensuring even clamping force is applied to both ends of sample. The test strip is then stretched along length ways of the SMA sample (or distance between the two tester arms was $95 \mathrm{~mm}$ ).

During the tests a current was applied to the samples, the temperature of the samples would increase due to the Joule heating effect. The SMA strips would begin to recover to their original shape when they reached their phase changing temperature. As the ends of the samples were restricted by the sample holders, generated force was applied onto the sample holders, then detected by the load cell. Results recorded are.

Measured time series of the force and force per section area (i.e. stress) were time stamped with the corresponding differential video frames (DFs) and the extracted 150 RBM components from each of the DFs.

\section{Input and output for the Machine Learning modelling}

It was important to note that for this study we aimed to develop a single predictive system for any shape of the SMA, hence all pre-processed data from the various shapes were combined into a single data set. The aim was to test a generalisation capability to predict force and stress while employing computer vision and machine learning algorithm. The data set included 10500 related to the "Omega", 9000 related to the "Half circle", 9500 related to the "V", 7500 related to the "4-bend" and finally 8500 related to the "Wave" shaped body under experimentation. The amount of force was represented by a number between 1-12 as the measured values, whereas the amount of stress was represented by a number between 1-20 as the measured values. They were directly suitable to be utilised as class labels of the proposed machine learning based multiclass classification problem. Altogether the data set had 45000 data entry points after removing all repetitive DFs with $5 \%$ or less variance between two consecutive video frames.

The final data set had 45000 selected differential video frames (DFs), each of which represented by extracted 150 RBM components, along with an associated measured amount of force and stress collected during five sets of experiments on SMA bodies with five different shapes (Figure 1).

The dimension of the whole data set was 45000 rows $X 152$ columns, where the first 150 columns were representing 150 RBM components (each row representing each of the DFs) and the last two columns were representing the force and stress values associated with each of the DFs.

The machine learning algorithms were trained with RBM components as inputs with force and stress as training target. In this way, during testing and validation, a trained model was prepared to predict force 
and stress against a set of unknown inputs. The unknown inputs were the extracted RBM components from a portion of the selected DFs which were not a part of the training of the algorithms.

For the predictive modelling using machine learning algorithms, first 150 columns of RBM components were used as training and testing inputs. The last two columns with values of measured force and stress were used as training and testing learning targets. A schematic diagram of the data pre-processing and machine learning based training and testing paradigm has been described in the Figure 4 .

\section{Machine Learning modelling}

Idea behind the quantization of the force and stress time series into multi-class representation was to simplify the quantification of the prediction accuracy in a traditional manner. It was also found that machine learning algorithms can be trained more accurately with coded class labels. This was the rationale behind the decision to take the multi-class classification over a traditional regression approach. We found that the multi-class coded representation of a continuous time series was best suited for the machine learning algorithms to learn and predict, while only inputs for the training and testing were the extracted 150 RBM components. In this approach, each of the selected DFs represented by a set of 150 RBM components, only needed two simple class labels as training targets, one for the associated force values and the other for the associated stress values, for the machine learning modelling.

The physically measured force of the 5 shapes at a specific time of the experiment was marked as the ground truth force associated with that DF to be learned as a target in the machine learning algorithm. The measured force timeseries were categorised into 12 force classes (e.g., 0-1, 1-2, 2-3..., 11-12) while each class represented a force range 1 Newton gradient) to formulate a quantisation based multiclass classification problem. Similarly, measured stress timeseries were categorised into 20 force classes (e.g., $0-1,1-2,2-3 . . ., 19-20)$, while each class represented a class of stress of range $1 \mathrm{MPa}$ gradient).

The development of the predictive model was based on a multi-output multi-class classification model. With suitable training the same feature space representing the changes in the video frames, a model was able to predict two class labels simultaneously, one for the force (class number ranging between 1-12) and other for the resistance stress (class number ranging between 1-20).

Because there are many possible types of machine learning classifiers, we tried ten types of classifier systems representing a wide range of algorithms. This was aimed to determine the most appropriate and efficient of these classifiers, and to justify the effectiveness of proposed machine learning framework for this study. As a comparative paradigm, namely, Feed Forward Back Propagation, Support Vector Machine, Multi-Layer Perception, Random Forest, Radial Basis Neural Network, Decision Tree, Naive Bayes, Quadratic Discriminant Analysis, Gradient Boosting, Logistic Regression classifiers were applied to the same data sets to establish the best artificially intelligent feature learning architecture as indicated by force and stress estimation accuracy [40-43]. 
Initially the whole data set was randomly split into $80 \%-20 \%$ proportional ten combinations. In each of the new subsets, $80 \%$ of the data (combination of inputs and targets) was used in the training-testing of the predictive model, while the rest of the $20 \%$ of the data was kept separately for a final validation of the trained model. In each of the ten training-testing phases with the $80 \%$ of the data, internally, a randomized 10-fold cross-validation (CV) technique was adapted to overcome the overfitting. In this well accepted approach in the machine learning domain, called $\mathrm{k}$-fold $\mathrm{CV}$, the training set is split into $\mathrm{k}$ smaller sets. The performance measure reported by $\mathrm{k}$-fold cross-validation is then the average of the values computed in the loop. This approach can be computationally expensive but does not waste too much data. Similarly, in each of the ten corresponding validations, mean accuracy was computed to report as a final accuracy estimation from the whole train-test-validate paradigm (Table 1).

\section{Results}

We can conclude that there are two main reasons for the superior classification performance of the Random Forest (RF) technique compared to Feed Forward Back Propagation, Support Vector Machine, Multi-Layer Perception, Radial Basis Neural Network, Decision Tree, Naive Bayes, Quadratic Discriminant Analysis, Gradient Boosting, and Logistic Regression [43-47]. RF classifier can adapt themselves to the distribution of abstract feature space extracted from a video stream, making them hugely popular as a robust classifier for various applications. RF was able to classify most of the patterns corresponding to force and stress classes, due to their ability to adjust their scale of generalization to match the morphological variability of the patterns in the video frames. RBM based feature extracted from the video frames helped to increase the linear separability of the multiclass classification problem. In Table 1, we also compared results between two scenarios, with and without using RBM features. In the later scenario whole flattened video frame was used as the input vector for the classifying task. It was evident that all the classifiers performed significantly better with RBM features while RF was outperforming other classifiers with an enhanced performance from $84 \%$ to $93 \%$ improved accuracy.

The performance of the estimators was quantified by the prediction accuracy ((TP + TN) / (TP + FN + FP $+T N)$ where $T P=$ true positives, $T N=$ true negatives, $F P=$ false positives, $F N=$ false negatives $)$. From the achieved mean accuracy from the 10-fold CV process, it was found that RNN was the best to handle this classification problem with $93 \%$ global accuracy with very low of false negatives. Results for the independent testing and validation are summarised in the Table 2 (for the generated force) and Table 3 (for the resistance stress).

Prediction accuracy, precision (defined by TP / (TP + FP)), recall (TP / $(T P+F N)$ ), F1-score (defined by 2 * (precision * recall) $/($ precision + recall)) and classification probability results are reported in these tables. The precision, recall, and F1 score (also F-score or F-measure) are measures of an experiment's accuracy, where a score of 1.0 indicates perfect classification and a score of 0.0 indicates all examples were incorrectly classified. Table 2 and Table 3 shows the complete classification report for the RF based multiclass classification results. Macro and Weighted average of the class-based prediction accuracy were also reported in the Table 2 and Table 3. 


\section{Conclusion}

Future generations of SMA materials will be designed with highly bio-inspired motivations. They will aim to be soft bodies composed of soft materials, part of soft actuators and sensors, and will be capable of soft movements and safe interaction with humans. Development and wider adaptation of SMA materials will rely on higher degree of freedom in movement and actuation.

In this work we aimed to develop a group of novel SMA materials suitable for large degree reversable actuation mechanism. We demonstrated that a newly developed Ti-Ni based SMA with equilibrium microstructure was able to achieve two-way actuation performance. Validation of this development was tested through consistent reversable actuation of various shapes of the same SMA material.

Through various experimental paradigm, we identified that there is a need to employ an intelligent system to characterise this novel material in order to achieve a greater control and intended actuation. Novel SMA material can only achieve a greater milestone in real life application, if combined with a suitable pathway to flexible actuation. It was established that a data driven framework is needed to rapidly estimate force and stress generated by a moving SMA body.

We reported accurate estimation of generated force and stress through an intelligent system instead of a conventional time-consuming approach. We combined computer vision techniques and scalable machine learning to develop a model-based architecture for rapid characterization of shape memory materials. The reported intelligent system was proven to be highly accurate in computer vision based rapid estimation of force and stress generated by a movement. Using computer vision and machine learning based modelling techniques to model deformable SMA bodies is a unique paradigm shift in the conventional characterisation of novel materials, which is predominantly actuated and controlled by central processing units and motors.

\section{Declarations}

\section{Author contributions}

RD, DR, CC and DL conceived the idea, did experimentation and machine learning modelling. They also contributed to the writing of the manuscript.

\section{Declaration of competing interest}

The authors declare that they have no known competing financial interests or personal relationships that could have appeared to influence the work reported in this paper.

\section{Acknowledgments}

Research was supported by CSIRO Interchange Program 2020, DATA61 Business Unit of CSIRO, Australia and Active Integrated Matter, a Future Science Platform at the CSIRO, Australia. The Authors would like to 
acknowledge Dr Kun Yang for technical clarification during manuscript revision.

\section{References}

1. Jani, J.M., Leary, M., Subic, A. Shape Memory Alloys in Automotive Applications. Applied Mechanics and Materials, 663, pp. 248-253 (2014).

2. Sun, J., Guan, Q., Liu, Y., \& Leng, J. Morphing aircraft based on smart materials and structures: A state-of-the-art review. Journal of Intelligent Material Systems and Structures, 27, pp. 2289-2312 (2016).

3. Jani J. M., Leary M., Subic A., Gibson M.A., A review of shape memory alloy research, applications and opportunities. Materials \& Design (1980-2015), 56, pp. 1078-1113 (2014).

4. Namrata Gangil, Arshad Noor Siddiquee, Sachin Maheshwari, Towards applications, processing and advancements in shape memory alloy and its composites, Journal of Manufacturing Processes, 59, pp. 205-222 (2020).

5. Wu Y., et al. Insect-scale fast moving and ultrarobust soft robot. Science Robotics, 4, eaax1594 (2019).

6. Paik J.K., Wood R.J, A bidirectional shape memory alloy folding actuator, 2012, Smart Materials and Structures, 21, p. 065013 (2012).

7. Reddy R.A., Hinglajia D.D., Modi A., Prithivirajan V., Hiremath S. R., Sazawal P., Mahapatra D.R., Morphing Airfoil with Thermally Activated SMA Actuators, ISSS Journal of Micro and Smart Systems, 6, pp. 29-45 (2017)

8. Sun H., Luo J., Ren Z., Lu M., Nykypanchuk D., Mangla S., and Shi Y. Shape Memory Alloy Bimorph Microactuators by Lift-Off Process. Journal of Micro and Nano-Manufacturing, 8, p. 031003 (2020).

9. Costanza G., Tata M.E., Shape Memory Alloys for Aerospace, Recent Developments, and New Applications: A Short Review, Materials, 13, p. 156 (2020).

10. D Josephine Selvarani R., D Glory Rebekah S., Design Considerations for Shape Memory Alloy-Based Control Applications in Actuators: Fundamentals, Principles, Materials and Applications (ed. Inamuddin, Boddula R., Asiri A.M.) pp. 17-31 (Scrivener Publishing LLC, 2020)

11. Lahoz R., Puértolas J.A., Training and two-way shape memory in NiTi alloys: influence on thermal parameters, Journal of Alloys and Compounds, 381, pp. 130-136 (2004).

12. Hayrettin C., Karakoc O., Karaman I., Mabe J.H., Santamarta R., Pons J., Two way shape memory effect in NiTiHf high temperature shape memory alloy tubes, Acta Materialia, 163, pp. 1-13 (2019).

13. Fortini A., Merlin M., Rizzoni R., Marfia S., TWSME of a NiTi strip in free bending conditions: experimental and theoretical approach, Materials Science, Fracture and structural Integrity, 29, pp. 74-84 (2014).

14. Peffer A., Denoyer K., Fossness E., and Sciulli D. Development and transition of low-shock spacecraft release devices. In Proceedings of IEEE Aerospace Conference, 2000, vol. 4, pp. 277-284 
15. Likhachev V.A., Razov A.I., Cherniavsky A.G., Kravchenko Y.D., and Trusov S.N., Truss mounting in space by shape memory alloys, In Proceeding of the First International Conference on Shape Memory and supereleastic Technologies Asilomar Conference center, Pacific Grove, California, USA, 1994

16. Jamie K.P., Robert J.W., A bidirectional shape memory alloy folding actuator, Smart Materials and Structures, 21, p. 065013 (2012)

17. Carpenter B. Lyons J. EO-1 technology validation report: lightweight flexible solar array experiment. Technical report NASA Godard Space Flight Center, Greenbelt, MD, 8 August 2001.

18. Kellam M.E., Song G, and Liang D., "Low-cost Thin Titanium Shape Memory Alloy Foils by Planar Flow Casting", Aeromat 2015, 11-14 May 2015, Long Beach, USA.

19. Derby S., et al. Critical review of current trends in shape memory alloy actuators for intelligent robots. Industrial Robot: An International Journal (2007).

20. Grant D. and Hayward V., Variable structure control of shape memory alloy actuators. IEEE Control Systems Magazine, 17(3), pp.80-88 (1997).

21. Kadir M.R.A., Dewi D.E.O., Jamaludin M.N., Nafea M., Ali, M.S.M., A multi-segmented shape memory alloy-based actuator system for endoscopic applications. Sensors and Actuators A: Physical, 296, pp.92-100 (2019).

22. Wang Y., Zhang C., Wu Z., Gao W., Zhou M., A hopfield neural network-based Bouc-Wen model for magnetic shape memory alloy actuator. AIP Advances, 10(1), p.015212 (2020).

23. Fremond M., Shape memory alloy. In Shape memory alloys (pp. 1-68). Springer, Vienna (1996).

24. Krulevitch P., Lee A.P., Ramsey P.B., Trevino J.C., Hamilton J., Northrup M.A., Thin film shape memory alloy microactuators. Journal of microelectromechanical systems, 5(4), pp.270-282 (1996).

25. Zeng Z., et al., Wire and arc additive manufacturing of a Ni-rich NiTi shape memory alloy: Microstructure and mechanical properties. Additive Manufacturing, 32, p.101051 (2020).

26. Lee J.H., Chung Y.S. and Rodrigue H., Long shape memory alloy tendon-based soft robotic actuators and implementation as a soft gripper. Scientific reports, 9(1), pp.1-12 (2019).

27. Lee J.I., Tsuchiya K., Tasaki W., Oh H., Sawaguchi T., Murakami H., Hiroto T., Matsushita Y., Park E., A strategy of designing high-entropy alloys with high-temperature shape memory effect. Scientific Report 9, 13140 (2019).

28. Lee, J., Ikeda, Y. \& Tanaka, I. First-principles screening of structural properties of intermetallic compounds on martensitic transformation. npj Comput Mater 3, 52 (2017).

29. Alkan S., Sehitoglu H., Prediction of transformation stresses in NiTi shape memory alloy. Acta Materialia, 175, pp.182-195 (2019).

30. Yang, S., Omori, T., Wang, C. et al. A jumping shape memory alloy under heat. Sci Rep 6, 21754 (2016).

31. Nazarahari A., Canadinc D., Prediction of the NiTi shape memory alloy composition with the best corrosion resistance for dental applications utilizing artificial intelligence. Materials Chemistry and 
Physics, 258, p.123974 (2021).

32. Sutskever I., Hinton G.E., Taylor G.W., The recurrent temporal restricted boltzmann machine. Advances in neural information processing systems, 21, pp.1601-1608 (2008).

33. Chen H., Murray A.F., Continuous restricted Boltzmann machine with an implementable training algorithm. IEE Proceedings-Vision, Image and Signal Processing, 150(3), pp.153-158 (2003).

34. Larochelle H., Mandel M., Pascanu R., Bengio Y., Learning algorithms for the classification restricted boltzmann machine. The Journal of Machine Learning Research, 13(1), pp.643-669 (2012).

35. Fiore U., Palmieri F., Castiglione A., De Santis A., Network anomaly detection with the restricted Boltzmann machine. Neurocomputing, 122, pp.13-23 (2013).

36. Marlin B., Swersky K., Chen B., Freitas N., March. Inductive principles for restricted Boltzmann machine learning. In Proceedings of the Thirteenth International Conference on Artificial Intelligence and Statistics, pp. 509-516 (2010).

37. Pirmoradi S., Teshnehlab M., Zarghami N., Sharifi A., The self-organizing restricted boltzmann machine for deep representation with the application on classification problems. Expert Systems with Applications, 149, p.113286 (2020).

38. Manukian, H., Pei, Y.R., Bearden, S.R.B. et al. Mode-assisted unsupervised learning of restricted Boltzmann machines. Commun Phys 3, 105 (2020).

39. Mocanu, D.C., Mocanu, E., Stone, P. et al. Scalable training of artificial neural networks with adaptive sparse connectivity inspired by network science. Nat Commun 9, 2383 (2018).

40. Carleo G., et al., Machine learning and the physical sciences. Reviews of Modern Physics, 91(4), p.045002 (2019).

41. Wei J., et al., Machine learning in materials science. InfoMat, 1(3), pp.338-358 (2019).

42. Schmidt J., Marques M.R., Botti S., Marques M.A., Recent advances and applications of machine learning in solid-state materials science. npj Computational Materials, 5(1), pp.1-36 (2019).

43. Tkatchenko A., 2020. Machine learning for chemical discovery. Nature Communications, 11(1), pp.14 (2020).

44. Hajiramezanali E., et al., Variational graph recurrent neural networks. In Advances in neural information processing systems, pp. 10701-10711 (2019).

45. Hang R., Liu Q., Hong D., Ghamisi P., Cascaded recurrent neural networks for hyperspectral image classification. IEEE Transactions on Geoscience and Remote Sensing, 57(8), pp.5384-5394 (2019).

46. Lyu F., Wu Q., Hu F., Wu Q., Tan M., Attend and imagine: Multi-label image classification with visual attention and recurrent neural networks. IEEE Transactions on Multimedia, 21(8), pp.1971-1981 (2019).

47. Graves A., Fernández S., Schmidhuber J., September. Multi-dimensional recurrent neural networks. In International conference on artificial neural networks, pp. 549-558 (2007).

\section{Tables}


Table 1 Multioutput Multiclass classification results from the ten different machine learning classifiers. A Random Forest (RF) classifier was the best performing classifier in the multiclass classification problem while predicting the generated force and the stress by a movement of a SMA body. It was found that a Restricted Boltzmann Machine (RBM) was able to extract features efficiently to enhance the prediction accuracies of the classifiers. These findings led to a predictive system which can help to control and actuate the SMA structures more accurately. Multioutput Multiclass Classifiers to
predict categories representing class of
generated force and resistance stress

\begin{tabular}{|c|c|c|c|}
\hline & $\begin{array}{l}\text { 10-Fold CV Mean } \\
\text { Test Accuracy } \\
\text { (\%) (+/- standard } \\
\text { deviation) }\end{array}$ & $\begin{array}{l}\text { Mean } \\
\text { Validation } \\
\text { Accuracy } \\
(\%) \\
(+/- \\
\text { standard } \\
\text { deviation) }\end{array}$ & $\begin{array}{l}\text { Mean Validation } \\
\text { Accuracy (\%) } \\
(+/- \text { standard } \\
\text { deviation) }\end{array}$ \\
\hline Feed Forward Back Propagation & $66.67(+/-11.56)$ & $\begin{array}{l}62.07(+/- \\
13.56)\end{array}$ & $52.07(+/-18.76)$ \\
\hline Support Vector Machine & $73.33(+/-10.07)$ & $\begin{array}{l}78.33(+/- \\
15.07)\end{array}$ & $70.21(+/-17.34)$ \\
\hline Multi-Layer Perception & $76.66(+/-15.6)$ & $\begin{array}{l}72.66(+/- \\
11.6)\end{array}$ & $73.66(+/-15.2)$ \\
\hline Radial Basis Neural Network & $89.11(+/-8.31)$ & $\begin{array}{l}86.56(+/- \\
8.31)\end{array}$ & $79.21(+/-11.91)$ \\
\hline Random Forest & $90.37(+/-5.07)$ & $\begin{array}{l}92.88(+/- \\
6.77)\end{array}$ & $84.18(+/-9.06)$ \\
\hline Decision Tree & $87.77(+/-17.77)$ & $\begin{array}{l}80.17(+/- \\
7.77)\end{array}$ & $73.17(+/-12.77)$ \\
\hline Naive Bayes & $70.44(+/-10.09)$ & $\begin{array}{l}71.44(+/- \\
14.09)\end{array}$ & $60.74(+/-13.79)$ \\
\hline Quadratic Discriminant Analysis & $60.00(+/-18.32)$ & $\begin{array}{l}65.00(+/- \\
16.32)\end{array}$ & $55.00(+/-18.30)$ \\
\hline Gradient Boosting & $75.55(+/-11.16)$ & $\begin{array}{l}73.15(+/- \\
12.16)\end{array}$ & $62.56(+/-15.36)$ \\
\hline Logistic Regression & $77.05(+/-12.87)$ & $\begin{array}{l}70.55(+/- \\
14.87)\end{array}$ & $67.55(+/-10.47)$ \\
\hline
\end{tabular}

Table 2 The complete classification report for the RF based multiclass classification results including prediction accuracy, precision, recall, F1-score of classification results from the independent validation 
stage while predicting class labels related to the classes of generated force by the SMA body movements.

\begin{tabular}{|lllll|}
\hline $\begin{array}{l}\text { Force class labels - equivalent } \\
\text { of generated measured force }\end{array}$ & $\begin{array}{l}\text { Precision } \\
\text { Estimation }\end{array}$ & $\begin{array}{l}\text { Calculated } \\
\text { F1-score }\end{array}$ & $\begin{array}{l}\text { Total number of support } \\
\text { samples for the } \\
\text { individual class }\end{array}$ \\
& & & & \\
\hline 1 & & & & \\
\hline 2 & 0.93 & 0.98 & 0.95 & 1268 \\
\hline 3 & 0.96 & 0.90 & 0.93 & 960 \\
\hline 4 & 0.92 & 0.89 & 0.91 & 608 \\
\hline 5 & 0.94 & 0.83 & 0.88 & 670 \\
\hline 6 & 0.90 & 0.89 & 0.89 & 540 \\
\hline 7 & 0.81 & 0.92 & 0.86 & 580 \\
\hline 8 & 0.84 & 0.88 & 0.86 & 900 \\
\hline 9 & 0.96 & 0.95 & 0.96 & 560 \\
\hline 10 & 0.99 & 0.97 & 0.98 & 506 \\
\hline 11 & 0.83 & 0.71 & 0.77 & 558 \\
\hline 12 & 1.00 & 0.85 & 0.92 & 890 \\
\hline Accuracy & 0.99 & 0.98 & 0.95 & 960 \\
\hline Wacro Avg & 0.91 & 0.89 & 0.90 & 9000 \\
\hline & 0.93 & 0.93 & 9000 \\
\hline
\end{tabular}

Table 3 The complete classification report for the RF based multiclass classification results including prediction accuracy, precision, recall, F1-score of classification results from the independent validation stage while predicting class labels related to the classes of stress. 


\begin{tabular}{|c|c|c|c|c|}
\hline $\begin{array}{l}\text { Stress class labels - } \\
\text { equivalent of generated } \\
\text { stress }\end{array}$ & $\begin{array}{l}\text { Precision } \\
\text { Estimation }\end{array}$ & $\begin{array}{l}\text { Recall } \\
\text { Estimation }\end{array}$ & $\begin{array}{l}\text { Calculated } \\
\text { F1-score }\end{array}$ & $\begin{array}{l}\text { Total number of support } \\
\text { samples for the individual } \\
\text { class }\end{array}$ \\
\hline 1 & 0.93 & 0.98 & 0.95 & 789 \\
\hline 2 & 0.96 & 0.90 & 0.93 & 678 \\
\hline 3 & 0.92 & 0.89 & 0.91 & 245 \\
\hline 4 & 0.94 & 0.83 & 0.88 & 376 \\
\hline 5 & 0.90 & 0.89 & 0.89 & 560 \\
\hline 6 & 0.81 & 0.92 & 0.86 & 344 \\
\hline 7 & 0.84 & 0.88 & 0.86 & 691 \\
\hline 8 & 0.96 & 0.95 & 0.96 & 230 \\
\hline 9 & 0.99 & 0.97 & 0.98 & 278 \\
\hline 10 & 0.83 & 0.91 & 0.92 & 501 \\
\hline 11 & 1.00 & 0.85 & 0.92 & 457 \\
\hline 12 & 0.99 & 0.98 & 0.95 & 890 \\
\hline 13 & 0.90 & 0.89 & 0.89 & 751 \\
\hline 14 & 0.81 & 0.92 & 0.86 & 220 \\
\hline 15 & 0.84 & 0.88 & 0.86 & 490 \\
\hline 16 & 0.96 & 0.95 & 0.96 & 600 \\
\hline 17 & 0.99 & 0.97 & 0.98 & 210 \\
\hline 18 & 0.90 & 0.89 & 0.89 & 201 \\
\hline 19 & 1.00 & 0.85 & 0.92 & 230 \\
\hline 20 & 0.99 & 0.98 & 0.95 & 259 \\
\hline Accuracy & & & 0.92 & 9000 \\
\hline Macro Avg & 0.92 & 0.88 & 0.91 & 9000 \\
\hline Weighted Avg & 0.91 & 0.92 & 0.92 & 9000 \\
\hline
\end{tabular}


Figures

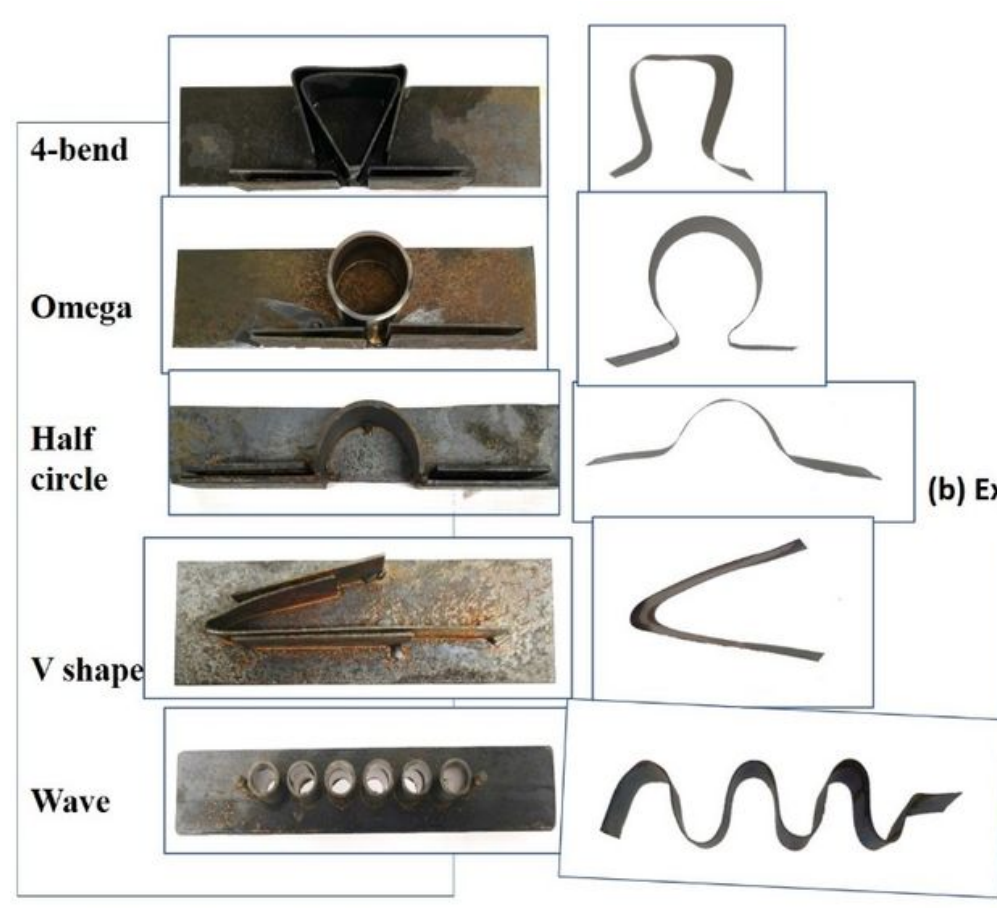

(a) Moulds and the shapes of the SMA produced by the moulds

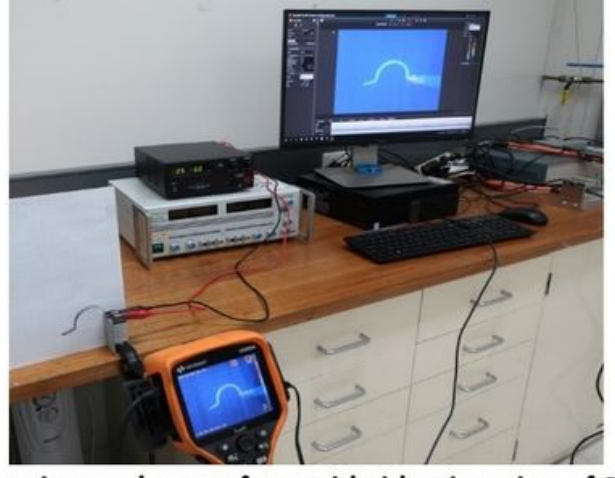

Experimental setup for rapid video imaging of SMA bodies

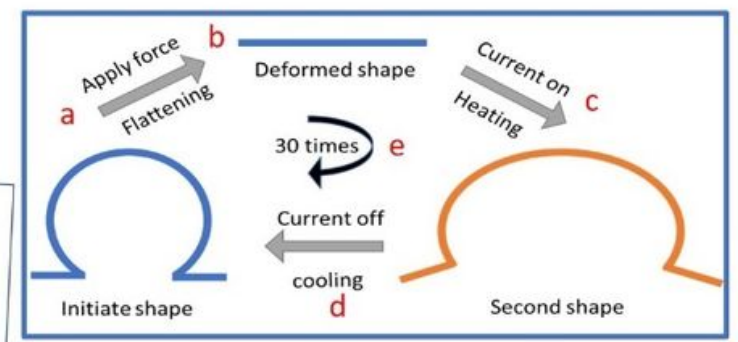

(c) Two-way shape memory effect training process

\section{Figure 1}

(a) Five steel moulds to shape SMA strips into different shapes and the shaped SMA strips in "Omega", "Half circle", "V", "4-bend" and "Wave" shapes; (b) Experimental setup to generate data for the rapid computer vision approach and machine learning based prediction; (c) Two-way shape memory effect training process, involving steps marked as a-e to train the body. 

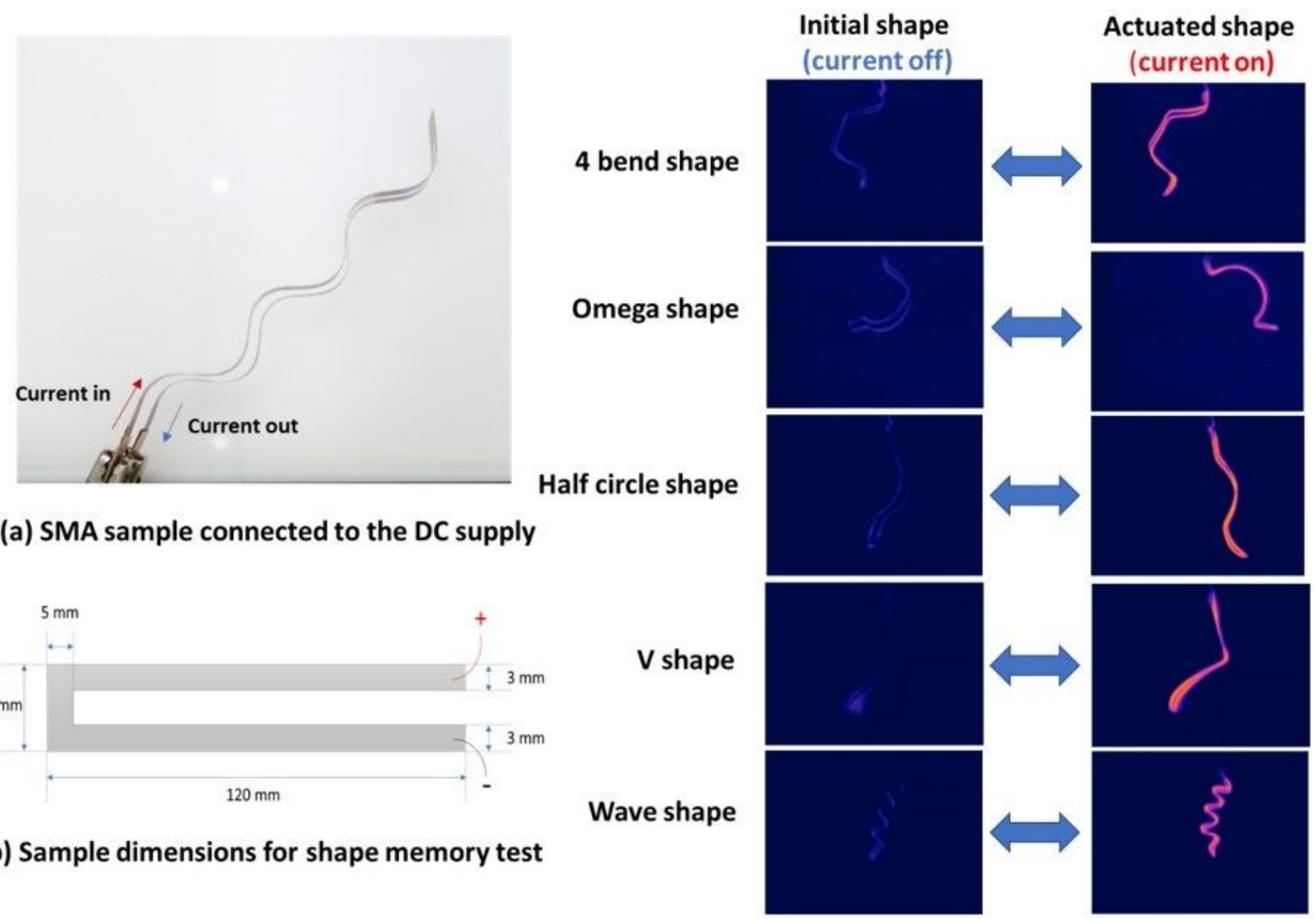

(a) SMA sample connected to the DC supply

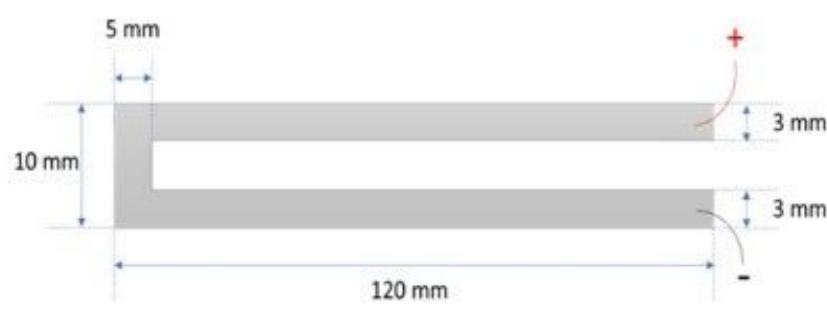

(b) Sample dimensions for shape memory test

(c) Shape memory effect of different shapes

Figure 2

(a) Two legs of SMA connected to the DC supply; (b) the sample dimension of SMA used in this study; (3) thermal images of the initial and actuated shape of the five different SMA bodies that were used in this study. The changes in shape and position during electrical excitement were the key dynamic information captured through the video imaging. The captured information was correlated with the measured force and stress to develop a predictive machine learning model. 


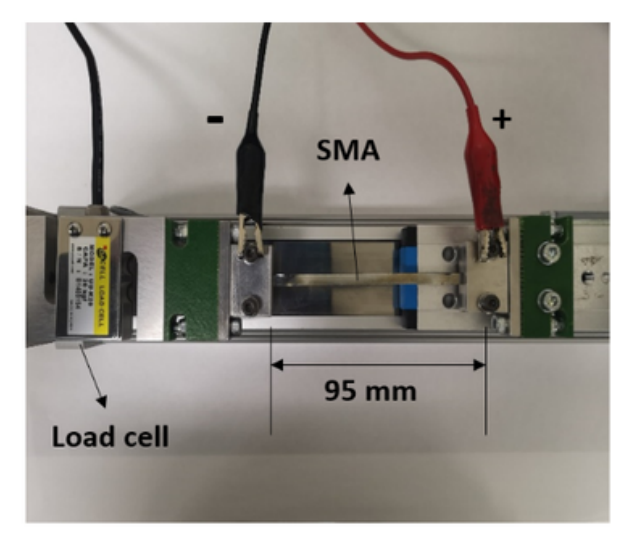

(a) Setup for force measurement (top-view)

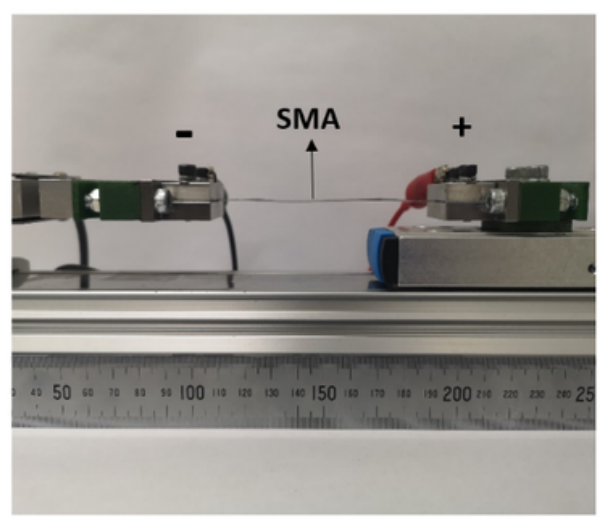

(b) Setup for force measurement (side-view)
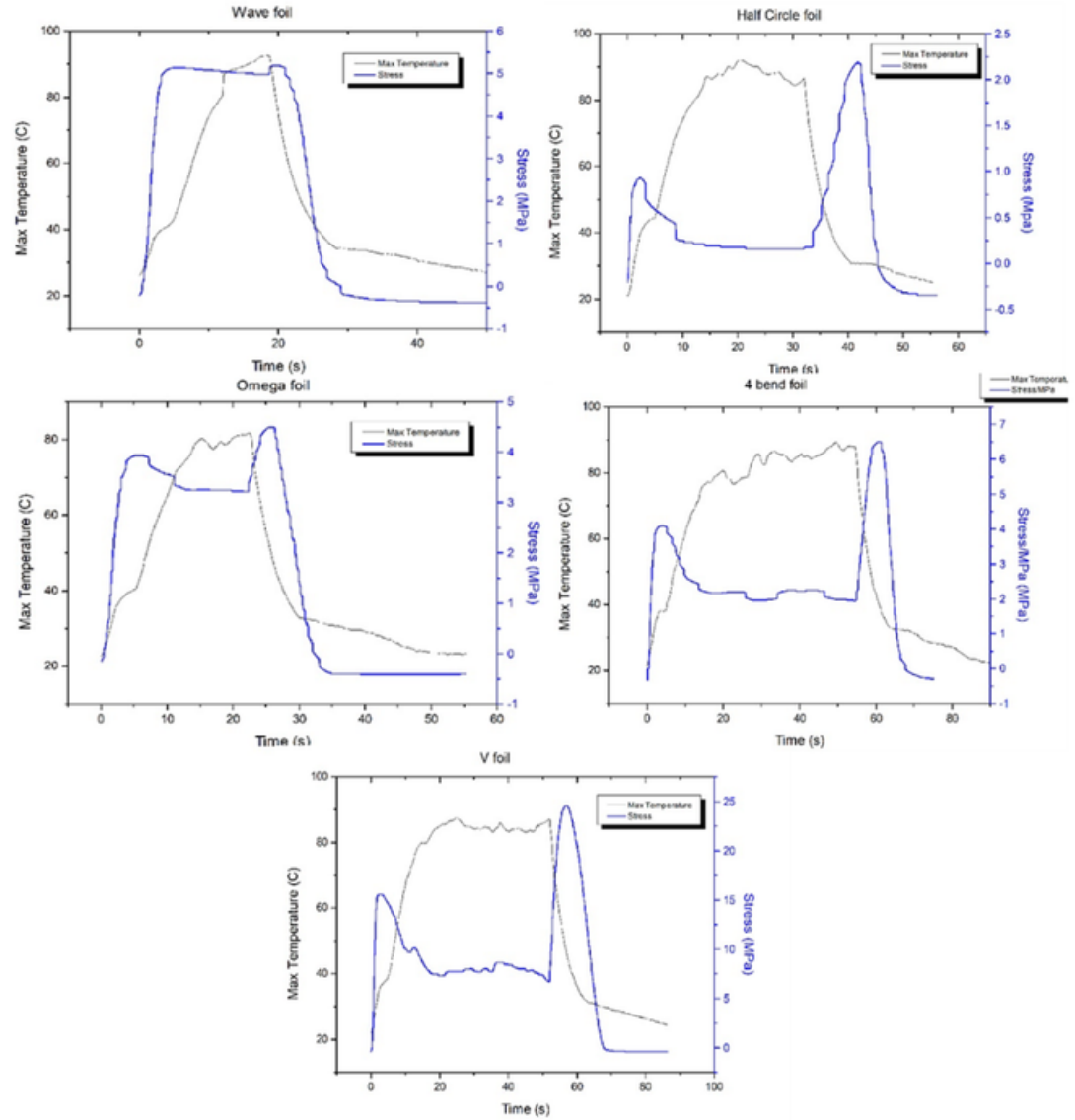

(c) Stress and temperature vs time curves of the SMAs in different shapes

\section{Figure 3}

Setup of the force measurement (a) top-view and (b) side-view. (c) shows the stress and temperature characteristics curves for the five SMA shapes. 


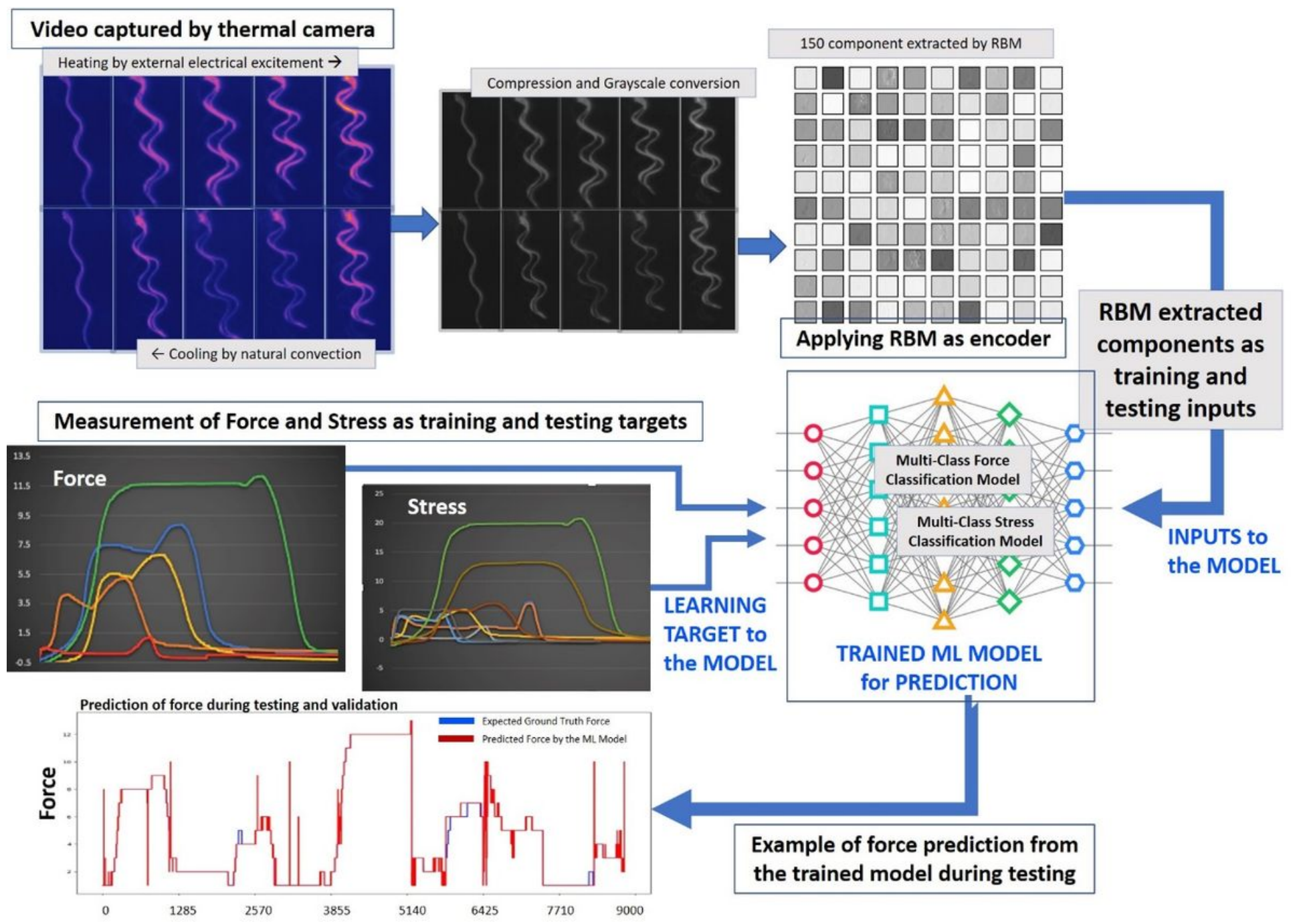

Figure 4

This figure shows the overall computer vision and machine learning based framework that was employed in this study. RBM based feature extraction combined with Random forest classification algorithm was able to improve the force and stress prediction accuracy from $84 \%$ to $93 \%$. This framework also demonstrated that a computer vision approach could be used to characterise novel material rapidly. 